\title{
Need for Speed: The Importance of Physiological Strain Rates in Determining Myocardial Stiffness
}

\author{
Matthew A. Caporizzo and Benjamin L. Prosser* \\ Department of Physiology, Perelman School of Medicine, The University of Pennsylvania, Philadelphia, PA, United States
}

The heart is viscoelastic, meaning its compliance is inversely proportional to the speed at which it stretches. During diastolic filling, the left ventricle rapidly expands at rates where viscoelastic forces impact ventricular compliance. In heart disease, myocardial viscoelasticity is often increased and can directly impede diastolic filling to reduce cardiac output. Thus, treatments that reduce myocardial viscoelasticity may provide benefit in heart failure, particularly for patients with diastolic heart failure. Yet, many experimental techniques either cannot or do not characterize myocardial viscoelasticity, and our understanding of the molecular regulators of viscoelasticity and its impact on cardiac performance is lacking. Much of this may stem from a reliance on

OPEN ACCESS

Edited by: Vasco Sequeira, University Hospital Würzburg,

Germany

Reviewed by:

Charles S. Chung,

Wayne State University, United States

Kenneth Scott Campbell,

University of Kentucky, United States

${ }^{*}$ Correspondence:

Benjamin L. Prosser

bpros@pennmedicine.upenn.edu

Specialty section:

This article was submitted to

Striated Muscle Physiology, a section of the journal

Frontiers in Physiology

Received: 17 April 2021

Accepted: 06 July 2021

Published: 30 July 2021

Citation:

Caporizzo MA and Prosser BL

(2021) Need for Speed:

The Importance of Physiological

Strain Rates in Determining

Myocardial Stiffness.

Front. Physiol. 12:696694. doi: 10.3389/fphys.2021.696694 techniques that either do not interrogate viscoelasticity (i.e., use non-physiological rates of strain) or techniques that compromise elements that contribute to viscoelasticity (i.e., skinned or permeabilized muscle preparations that compromise cytoskeletal integrity). Clinically, cardiac viscoelastic characterization is challenging, requiring the addition of strain-rate modulation during invasive hemodynamics. Despite these challenges, data continues to emerge demonstrating a meaningful contribution of viscoelasticity to cardiac physiology and pathology, and thus innovative approaches to characterize viscoelasticity stand to illuminate fundamental properties of myocardial mechanics and facilitate the development of novel therapeutic strategies.

Keywords: viscoelasticity, diastole, diastolic dysfunction, HFPEF, myocardial compliance, cardiac mechanics

\section{INTRODUCTION}

\section{Cardiac Viscoelasticity in Health and Disease}

Reduced cardiac compliance is associated with aging and broadly observed across multiple etiologies of heart failure (Chen et al., 1998; Zile and Brutsaert, 2002; Kass, 2005; Borlaug et al., 2013b). Stiffening arises from remodeling of the extracellular matrix (van Heerebeek et al., 2008; Chris and Jacobs, 2017; Frangogiannis, 2019), changes to the myocyte cytoskeleton (Cooper, 2006; Sequeira et al., 2014; Chen et al., 2018), and modifications to the sarcomere (Miyata et al., 2000; Le Winter and Van Buren, 2002; van der Velden, 2011; Hamdani et al., 2013; Zile et al., 2015). Since myocardium is viscoelastic, its compliance decreases with increasing rate of strain (Rankin et al., 1977; Harris et al., 2002; Wang et al., 2016), and thus proper measurement of myocardial compliance requires physiological loading conditions. Common assessments of myocardial mechanics utilize highly variable loading conditions, resulting in different interpretations about the role of viscoelasticity in cardiac function (Brutsaert et al., 1971; 
Liu and Wang, 2019). As such, viscoelasticity remains an understudied and poorly understood property of the heart.

The viscoelasticity of the myocardium is an important determinant of cardiac performance in health and disease (Rankin et al., 1977; Wang et al., 2016). Viscous forces influence the rate of myocardial shortening (de Tombe and ter Keurs, 1992), and as the heart fills with blood in diastole, the stretching of the left ventricle against diastolic blood pressure invokes considerable viscoelastic forces (Hess et al., 1979; Caporizzo et al., 2020). This viscoelasticity arises from the slip of cytoskeletal and extracellular cross links that dissipate energy (Nam et al., 2016), and the force reached prior to bond slip, and therefore the stiffness of myocardium, depends strongly on the rate of stretching (Stroud et al., 2002; Linke and Leake, 2004; Nishimura et al., 2006; Caporizzo et al., 2020). Consequently, stressors such as exercise, which increase heart rate and accelerate diastolic filling, amplify viscoelastic forces (Tsutsui et al., 1993; Fraites Thomas et al., 1997; Kass et al., 2004). With viscoelasticity notably greater in heart disease (Harris et al., 2002; Caporizzo et al., 2020), it is not surprising that exercise intolerance is an early symptom for these patients. Still, our understanding of myocardial viscoelasticity and its regulation in the context of stressors and pathophysiology remains incomplete.

\section{Viscoelasticity and Cardiac Output: Implications for Myocardial Reserve}

Controlling myocardial viscoelasticity may be particularly consequential for maintaining myocardial reserve, the ability of the heart to increase its power output in response to increased demand. Viscous forces proportionally increase ventricular stiffness by the rate of ventricular filling. During exercise, reserve capacity is utilized by increasing the heart rate while maintaining the stroke volume (Barmeyer et al., 2009; Borlaug et al., 2013a). As heart rate increases, so does the filling rate (Smiseth, 2018), creating a condition where elevated viscoelasticity may limit filling during exercise.

Figure 1 illustrates the cardiac work loop and how viscoelasticity changes the shape of the diastolic pressure-volume relationship as filling rate changes. The work of each beat is represented by a plot of ventricular pressure vs. volume forming a "work loop" where the area is the stroke work (Figure 1A). The bottom of the loop represents the pressure and volume increase during diastolic filling, with the slope at any point along the diastolic curve representing the effective stiffness of the left ventricle at that point of diastole (diastolic filling: bold segment Figure 1A). At a resting human heart rate of 60 beats per minute, diastole occurs over about $600 \mathrm{~ms}$ divided into less than $100 \mathrm{~ms}$ for isovolumic relaxation (downward arrow) and about $500 \mathrm{~ms}$ for ventricular filling. The majority of ventricular filling occurs during two intervals of rapid stretching early and late in diastole over which the myocardium strains by $10-20 \%$ in about $200 \mathrm{~ms}$ separated by a period of diastasis (Smiseth, 2018). Thus, diastole invokes viscous forces from the myocardium and the stiffness of the myocardium depends on the filling rate. In the absence of compensating factors, as the heart rate increases under stress, viscoelastic forces impair filling

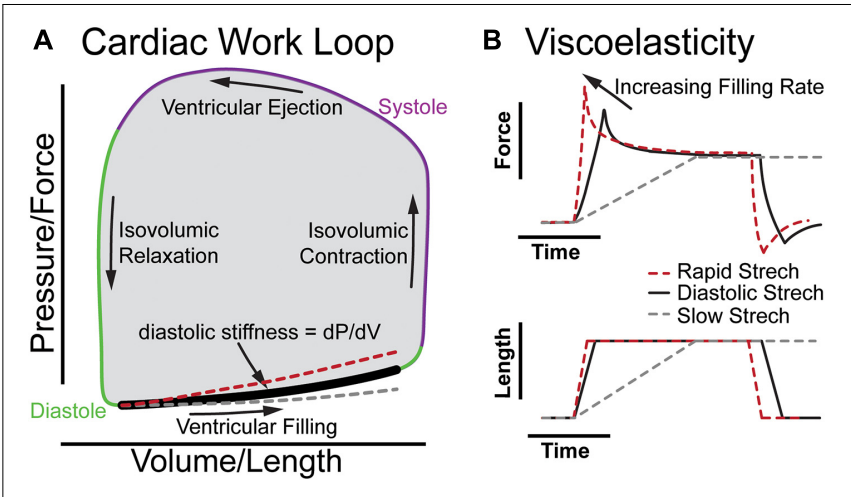

FIGURE 1 | The role of viscoelasticity in determining diastolic compliance. (A) Illustration of cardiac work loop with diastolic filling and effective stiffness. The red and gray curves indicate increased or decreased filling rate, respectively. (B) The associated force vs. time and length vs. time plots for a diastolic stretch and an isometric hold to reveal viscoelastic relaxation.

by increasing the diastolic pressure volume relationship (red dashed line Figure 1A), which would reduce stroke volume, and cardiac output to limit myocardial reserve. In healthy individuals, the beta-adrenergic response may compensate for viscous forces (Fukuda et al., 2005; Falcão-Pires et al., 2011). However, consistent with elevated myocardial viscoelasticity limiting exercise tolerance, patients with diastolic heart failure often exhibit reduced end diastolic volume despite increased diastolic pressure during exercise (Barmeyer et al., 2009; Borlaug et al., 2013a). This underscores the importance of understanding the role of viscoelasticity in cardiac performance.

Figure 1B breaks down the time-dependence of a continuous diastolic stretch to more clearly illustrate the influence of myocardial viscoelasticity on ventricular compliance at different physiological strain rates. This is effectively a portion of the bold segment in 1A split into stress vs. time and volume vs. time. When the length is held constant, the viscoelasticity is evident by the stress relaxation (Figure 1B; Caporizzo et al., 2018). This behavior is not readily appreciated in a cardiac work loop where time is not represented. Yet, the difference between the stiffness at a physiological rate vs a slow rate of strain (dashed gray line) is considerable. Analogously, when the diastolic interval decreases and myocardium is stretched faster (dashed red line) viscoelasticity increases the effective stiffness (Harris et al., 2002; Caporizzo et al., 2018, 2020). On the work loop this behavior appears as a steeper slope of the diastolic pressure-volume curve (red dashed line), which can limit diastolic filling in cases of pathologically increased viscoelasticity (Koide et al., 2000). It should be noted that diastole is a complex multi-component process that is influenced by the lungs, circulatory system, and all four chambers of the heart (Yellin et al., 1992; Kovács et al., 2000), and that while here we use a constant strain-rate to illustrate viscoelastic principles in Figure 1, the left-ventricular myocardium fills at a variable rate in diastole with different phases exhibiting unique mechanical behavior that simple mechanical models alone do not fully capture (Zhang and Kovács, 2008). 


\section{The Cast of Characters: Viscoelasticity in the Myofilament, Myocyte, and Myocardium}

Myocardial compliance is regulated at the myofilament, cytoskeletal and extracellular level. At the molecular level tissue viscoelasticity arises from cross-links which break and reform when the material is stretched or compressed. There are numerous such instances of slip bonds in myocardium, such as actomyosin cross-bridges, cell-cell and cell-matrix interactions, and cytoskeletal cross-linking and anchoring complexes. Thus, viscoelasticity is a multi-scale problem with tissue level, myocyte level, and myofilament level contributors.

The myofilaments, which are responsible for the contractile capacity of cardiac tissue, have highly regulated mechanical behavior governed in large part by post-translational modifications and changes in sarcomere stoichiometry (Miyata et al., 2000; Hamdani et al., 2013; LeWinter and Palmer, 2015). The giant protein titin is considered to be a primary determinant of myofilament diastolic tension (Granzier and Irving, 1995) and a significant contributor to myofilament viscoelasticity (Chung et al., 2011). Titin compliance is regulated by phosphorylation, which is commonly altered in disease affecting the diastolic compliance of skinned myocardium (Hamdani et al., 2013; Herwig et al., 2020). Myosin binding protein C acts as a drag, i.e., viscous, element in the sarcomere, bridging the thick and thin filaments in the "C-zones", and is important for regulating force generation and relaxation (Palmer et al., 2004; Rosas et al., 2015). Myosin activation during systole is the primary determinant of cardiac systolic stiffness, i.e., force generation, although the contribution of cross-bridges to stiffness in the relaxed state remains somewhat uncertain and may depend on conditions (Sequeira et al., 2014). While regulation of myofilament contractile function has an established role in cardiac health and disease, the passive role of myofilament stiffness in regulating diastolic filling is an active area of research.

The non-sarcomeric cytoskeleton consisting of microtubules and desmin intermediate filaments is critical for sarcomere organization (Robison et al., 2016) and regulates myocyte viscoelasticity (Caporizzo et al., 2020). The viscoelastic contribution of microtubules requires their post translational detyrosination which promotes the binding of various microtubule associated proteins that stabilize the microtubule network and promote its cross-linking with the myocyte cytoskeleton and intermediate filament network (Cooper, 2006; Kerr et al., 2015; Chen et al., 2018). Desmin intermediate filaments are elastic elements in the myocyte that wrap around the myofilament Z-disk to maintain sarcomere registry. In heart failure, there is an increased abundance of both detyrosinated microtubules and desmin intermediate filaments (Chen et al., 2018). Together, the microtubule and desmin filament networks form a lattice-like scaffold that contributes to myocyte viscoelasticity facilitates sarcomere maintenance and organization (Caporizzo et al., 2019).

Deposition of extracellular matrix, i.e., fibrosis, is a common feature of dilated and ischemic heart failure in particular (Gulati et al., 2013; Perestrelo et al., 2021). When the ventricular wall is weakened by high filling pressures or ischemic insult, stiffening through fibrosis is necessary to prevent diastolic overstretching or even wall rupture (Nagai et al., 2014). Fibrosis can dramatically stiffen myocardium and is largely thought of as an irreversible feature of disease, as it is resistant to remodeling (Webber et al., 2020). Aside from fibrosis, the cardiac extracellular matrix is rich in viscoelastic proteoglycans that are linked to hypertrophic signaling and overexpressed in multiple etiologies of heart failure (Christensen et al., 2019). The basement membrane consists of type four collagens, laminins, nidogen and other specific ECM proteins which serve as the interface for cell surface receptors (Perestrelo et al., 2021). The overall composition of the ECM is altered in disease independent of fibrosis, but the influence composition has on myocardial viscoelasticity or myocyte remodeling remains unclear.

\section{In a Non-linear Viscoelastic Myocardium-What Is the Right Mechanical Test?}

Like most biological material, myocardium is a non-linear viscoelastic material (Humphrey et al., 1990; Harris et al., 2002; Linke and Leake, 2004), meaning that its stiffness depends on both the rate and magnitude by which it is stretched or compressed. In an intact heart, the sarcomere length typically ranges from about $1.8 \mu \mathrm{m}$ in systole to $2.1 \mu \mathrm{m}$ in diastole (Sonnenblick et al., 1967). Depending on the magnitude of strain, structural proteins such as microtubules, titin, and collagen become aligned and maximally extended, which sharply increases their contribution to stiffness. Filamentous elements in the myocardium typically run parallel to the contractile axis of the cardiomyocytes, which form an anisotropic crosshatched pattern in the ventricular free wall. When sarcomere length is extended beyond $2.1 \mathrm{um}$, the non-linear stiffening associated with fiber alignment of titin and collagen becomes the dominant factor determining myocardial stiffness (Linke and Leake, 2004; Zile et al., 2015; Caporizzo et al., 2020). This length-dependent stiffening is important to prevent the heart from becoming overstretched or injured but adds a degree of complexity in designing experiments to measure physiologically relevant viscoelastic properties.

In addition to non-linear mechanical properties, the heart expands during diastole, meaning that the myocytes are stretched along both their length and width which leads to compression along their axis normal to both the contractile axis and ventricular wall. Tensile tests only stretch the material in $1 \mathrm{D}$, while compressive tests do not typically stretch sarcomeres, as compression is commonly applied orthogonal to the contractile axis. 2D stretching assays are more physiologically relevant but are highly specialized and difficult to employ. The easiest physiological approximation of diastolic compliance likely comes from stretching myocytes or myocardium in 1D with $10-20 \%$ strain in about 100-200 ms along the contractile axis. At these rates myocardial stiffness is increased by about $30-50 \%$ by viscoelastic forces (Caporizzo et al., 2020).

The complexity of a non-linear, anisotropic, viscoelastic material makes it difficult to extrapolate physiological 
implications from a single measurement technique. Yet the use of orthogonal approaches, when combined with varying strain and loading rates and modeling, enables the contributions of sarcomeres, microtubules, and extracellular matrix to be isolated.

\section{The One Dimensional: Force-Length Relationship}

One dimensional mechanical tests are the most common form of measuring myocardial mechanics enabling assessment of both systolic and diastolic properties. Isometric force generation is a typical method to determine the contractile capacity of the heart, conducted by holding the muscle at constant length and introducing calcium to demembranated myocardium (Sequeira et al., 2014) or by electrically stimulating intact myocardium. Similarly, the elasticity of relaxed myocytes or myocardium can be determined by measuring the tension held at various sarcomere lengths. While "resting tension" is an important metric of diastolic stiffness, it only captures elasticity and is effectively a minimum value of stiffness. Tensile tests determine the stressstrain response and can be conducted at physiological strain rates where viscoelasticity is observed (Linke and Fernandez, 2003; Caporizzo et al., 2018). In these experiments, precise length control and force-feedback is possible and has been used to mimic the cardiac cycle to generate work loops in beating myocytes and myocardium (Helmes et al., 2016). These experiments clearly demonstrate that both active and passive myocardial mechanics depend on both the length (Chung, 2020) and the rate of shortening or lengthening (Sonnenblick, 1962; Brutsaert et al., 1971; Chung et al., 2017).

\section{Force Spectroscopy}

Scanning probe-based force spectroscopy employs micro and nanoscale cantilevers (Lieber et al., 2004; Lyon et al., 2009), fluid jets (Swiatlowska et al., 2020), and suction pipettes, to deform myocytes and myocardium to determine viscoelastic properties. Scanning probe microscopy offers the advantage of high spatial resolution that can resolve local stiffness differences between T-tubules, intercalated disks, Z-disks, and M-lines. Scanning nanojet microscopy has been employed for example to demonstrate that detyrosinated microtubules contribute significantly to both the structure and stiffness of the cardiomyocyte cortex at the costamere (Swiatlowska et al., 2020). Determining viscoelasticity requires making measurements at different strain rates or frequencies and modeling the ratedependence of the modulus. This can be done by either indenting at different speeds (Caporizzo et al., 2015) or by pressing on the material and then oscillating the probe in a low-amplitude frequency sweep to determine the elastic and viscous components of the stiffness (Grant et al., 2012). These experiments find that cardiomyocytes are best modeled by a standard linear solid with an additional parallel viscous element (Robison et al., 2016; Caporizzo et al., 2018). Various groups have successfully observed increased viscoelasticity of cardiomyocytes in heart disease with atomic force microscopy (Nishimura et al., 2006; Chen et al., 2018). However, it is

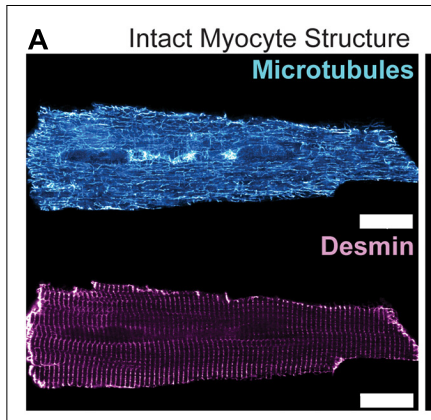

B Intact Myocyte Viscoelasticity
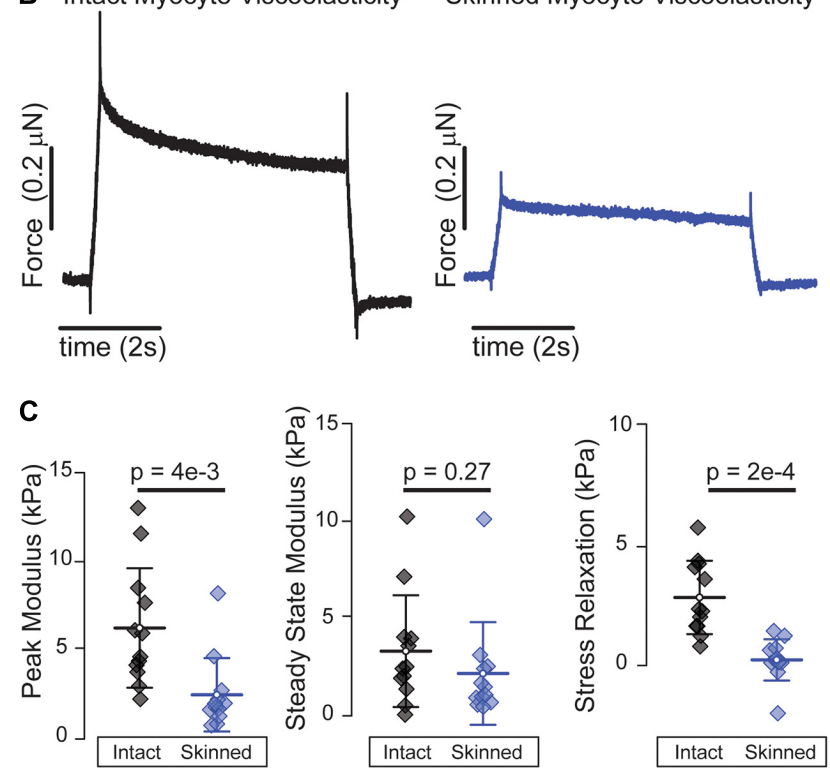

FIGURE 2 | Skinned cardiomyocytes exhibit a loss of microtubules and reduced viscoelasticity. (A) Representative fluorescence images of microtubules and desmin intermediate filaments in an intact (left) and skinned (0.1\% Triton X-100 30 min at room temperature) (right) cardiomyocyte. (B) Average viscoelasticity of intact cardiomyocytes (left) and skinned cardiomyocytes (right). (C) Average data for peak, steady-state and stress relaxation, mean line with $\mathrm{SD}$ whiskers; $p$-values determined from paired-sample $t$-test. $N=12$ rates and $n=12$ myocytes for each. Viscoelastic test is a 15\% length step in $200 \mathrm{~ms}$. Scale bar $=20 \mu \mathrm{m}$.

challenging to extrapolate compressive or cortical stiffness, where myocytes are compressed orthogonal to their contractile axis, to physiological tissue level mechanics, where myocytes are stretched along their contractile axis. These techniques also rely on smaller strains than are typically seen in the cardiac cycle, where larger viscoelastic contributions are observed. Nevertheless, it has unrivaled mechanical resolution, making it a go-to technique for determining local or element-specific stiffness of intact samples.

\section{In vivo Assessment of Viscoelasticity}

Echocardiography is routinely employed to measure chamber dynamics in patients as it allows for an easy and non-invasive assessment of cardiac systolic and diastolic function (HildickSmith, 2001). Echocardiography can be used to characterize 
diastolic dysfunction, but determining the underlying cause from imaging alone can be difficult (Nagueh, 2020). Quantitative determination of ventricular stiffness in vivo requires the simultaneous collection of chamber pressure and volume, as achieved using conductance catheter hemodynamic assesment (Ogilvie et al., 2020). Typically, during these procedures patients are anesthetized and their heart rate is constant. Thus, altering filling rate to assess viscoelasticity is impractical. Lifting patients' feet to transiently increase venous return is one way to accelerate LV filling (Elwan et al., 2018) and could enable an estimate of in vivo viscoelasticity. While crude, this type of procedure can provide insight into the rate-dependent mechanical response, and may provide useful information about a patient's exercise capacity and the severity of their underlying disease.

\section{Practical Considerations}

Many of the aforementioned mechanical interrogations are conducted in reduced preparations of myofibrils, myocytes, and myocardium at either room or physiological temperature. Both temperature (Templeton et al., 1974) and chemical permeabilization, i.e., "skinning," affect myocyte viscoelasticity (King et al., 2011). Microtubule polymerization dynamics are sensitive to temperature and while microtubules generally remain intact at room temperature, exposure to ice cold solutions as is common in myocardial preparations can depolymerize a significant fraction of microtubules (Fassett et al., 2019). Skinned preparations allow for robust attachment of myocytes via cytotoxic glues that enable repeated stretching beyond sarcomere lengths of $2.1 \mu \mathrm{m}$ which is difficult to achieve in intact preparations that rely on myocyte-friendly extracellular glues such as Myotak $^{\mathrm{TM}}$. Skinning leads to an expansion of the myofilament lattice that reduces diastolic stiffness at physiological (King et al., 2011) and room temperature (Figures 2B,C) which can be restored by adding high molecular weight polymers such as $2-4 \%$ dextran to solution (McDonald and Moss, 1995; King et al., 2011). Skinning also removes key viscoelastic elements such as membranes, microtubules (Figure 2A), and cytosolic proteins, which is reflected in the reduced viscoelasticity of skinned preparations as shown by a greater than proportional decrease in the stress relaxation of skinned myocytes, Figure 2C. "Gentler" permeabilization techniques such as alpha toxin and saponin have been employed to better retain membrane-bound proteins than typical ionic surfactants (Kuznetsov et al., 2008), but while these do retain membrane proteins, the loss of soluble cytosolic factors and the breakdown of structural elements such as the microtubules can still compromise viscoelasticity. Thus, while skinned myocytes represent a powerful and informative platform for interrogating myofilament mechanical properties, one needs to keep in mind that such reductionist approaches can significantly reduce the viscoelasticity of the cardiomyocyte.

\section{Concluding Remarks}

Overall, myocytes and myocardium exhibit non-linear viscoelasticity, meaning that ventricular mechanical properties depend on both the amount and speed they stretch. Myocardial viscoelasticity is a determinant of diastolic stiffness and likely influences cardiac exercise capacity. Ventricular viscoelasticity increases in heart failure due to multiscale remodeling of myofilaments, microtubules, and the extracellular matrix, and while much of these changes have been well characterized in end-stage heart failure, their role in the physiology of cardiac performance is an active area of research. Different viscoelastic characterization techniques provide meaningful information about different aspects of cardiac stiffness, but relating viscoelastic parameters to in vivo function remains a challenge. Directly assessing myocardial viscoelasticity in vivo also presents a challenge as it necessitates modifying venous return during hemodynamic assessment. Nevertheless, emerging research demonstrating a causal role of increased viscoelasticity in the pathology of heart failure motivates the development of new characterization techniques and strategies to manipulate this understudied component of cardiac mechanics.

\section{Experimental Methods}

Cell Stretch: Adult cardiomyocytes were isolated by Langendorff perfusion as described previously (Robison et al., 2016). Myocytes were then placed in culture in a $95 / 5$ incubator at $37^{\circ} \mathrm{C}$ in M199 media supplemented with $20 \mathrm{mM}$ HEPES pH 7.4, 1× Insulin Selenium and Transferrin (Thermo Scientific 51500056), $1 \mathrm{mg} / \mathrm{mL}$ Primocin (Invivogen), and $25 \mu \mathrm{M}$ Cytochalasin D (Cayman Chemical Company). Following incubation, cells were exchanged into room temperature normal Tyrode's solution (Robison et al., 2016) containing $30 \mathrm{mM} \mathrm{BDM}$ and attached to the Myostretcher Apparatus with Myotak (Ion Optix LLC). Intact myocytes were stretched via a $200 \mathrm{~ms}$ ramp and held at length for $5 \mathrm{~s}$ as shown in Figure 1B which corresponded to a stretch from an average sarcomere length of $1.9 \mu \mathrm{m}$ resting to $2.1 \mu \mathrm{m}$ stretched. Tyrode's was then exchanged for relaxing buffer (7.8 mM ATP, $20 \mathrm{mM}$ phosphocreatine, $20 \mathrm{mM}$ imidazole, $4 \mathrm{mM}$ EGTA, $12 \mathrm{mM} \mathrm{Mg}$-propionate, and $97.6 \mathrm{mM}$ K-propionate) and allowed to equilibrate for $5 \mathrm{~min}$ before relaxing buffer containing $0.1 \% \mathrm{~T} \times 100$ was introduced into the chamber. Myocytes were continuously observed using the eye piece and transmitted light on a Zeiss Axio Observer Z.1 inverted optical microscope with a Zeiss 1.4 NA $63 \times$ oil immersion objective for $30 \mathrm{~min}$ of demembranation at which point cell contrast was notably reduced but sarcomere length was still clearly visible. An identical stretch (in terms of resting to stretched sarcomere length) was conducted on the skinned myocyte to determine the skinned cell viscoelasticity. Peak, steady state, and stress-relaxation was quantified from the resulting stressstrain plots. Experiments were conducted at a room temperature of $\sim 22 \mathrm{C}$.

Immunofluorescence: Cardiomyocytes were gravity sedimented in media and resuspended in relaxing buffer containing $0.1 \% \mathrm{~T} \times 100$ for $25 \mathrm{~min}$ on a rocker before gravity sedimentation for the final 5 min of skinning. Supernatant was removed and cells were then immediately fixed in chilled methanol $\left(-20^{\circ} \mathrm{C}\right)$ for $7 \mathrm{~min}$. Following fixation, cells were exchanged into blocking buffer and incubated with primary 
antibodies for alpha tubulin (mouse DM1A-Cell Signaling Technology 1:500) and desmin (goat Y-20, Santa Cruz Biotechnology- 1:200) for $48 \mathrm{~h}$. Cells were then rinsed and exchanged into secondaries (Thermo Fisher Scientific, A10037 and Life Technologies, A21082) for $48 \mathrm{~h}$ of incubation. Cells were then pelleted and resuspended in Prolong Diamond antifade (Invitrogen) and mounted between and glass slide and coverslip for imaging.

Confocal imaging was conducted on a Zeiss 980 laser scanning confocal microscope equipped with Airyscan through a $63 \times$ oil 1.4 NA objective lens. Image analysis was performed using Zen Black for Airyscan processing and Image J was used for image preparation.

Unadjusted $p$-values were calculated using paired sample $t$-tests in Origin Lab 2019.

\section{DATA AVAILABILITY STATEMENT}

The raw data supporting the conclusions of this article will be made available by the authors, without undue reservation.

\section{REFERENCES}

Barmeyer, A., Müllerleile, K., Mortensen, K., and Meinertz, T. (2009). Diastolic dysfunction in exercise and its role for exercise capacity. Heart Fail Rev. 14, 125-134. doi: 10.1007/s10741-008-9105-y

Borlaug, B. A., Jaber, W. A., Ommen, S. R., Lam, C. S., Redfield, M. M., and Nishimura, R. A. (2013a). Diastolic relaxation and compliance reserve during dynamic exercise in heart failure with preserved ejection fraction. Heart 97, 964-969. doi: 10.1136/hrt.2010.212787

Borlaug, B. A., Redfield, M. M., Melenovsky, V., Kane, G. C., Karon, B. L., Jacobsen, S. J., et al. (2013b). Longitudinal changes in left ventricular stiffness. Circ. Heart Fail. 6, 944-952.

Brutsaert, D. L., Claes, V. A., and Sonnenblick, E. H. (1971). Velocity of shortening of unloaded heart muscle and the length-tension relation. Circ. Res. 29, 63-75. doi: 10.1161/01.RES.29.1.63

Caporizzo, M. A., Chen, C. Y., and Prosser, B. L. (2019). Cardiac microtubules in health and heart disease. Exp. Biol. Med. 9:153537021986896. doi: 10.1177/ 1535370219868960

Caporizzo, M. A., Chen, C. Y., Bedi, K., Margulies, K. B., and Prosser, B. L. (2020). Microtubules increase diastolic stiffness in failing human cardiomyocytes and myocardium. Circulation 141, 902-915. doi: 10.1161/CIRCULATIONAHA.119. 043930

Caporizzo, M. A., Chen, C. Y., Salomon, A. K., Margulies, K. B., and Prosser, B. L. (2018). Microtubules provide a viscoelastic resistance to myocyte motion. Biophys. J. 115, 1796-1807. doi: 10.1016/j.bpj.2018.09.019

Caporizzo, M. A., Roco, C. M., Ferrer, M. C. C., Grady, M. E., Parrish, E., Eckmann, D. M., et al. (2015). Strain-rate dependence of elastic modulus reveals silver nanoparticle induced cytotoxicity. Nanobiomedicine 2:9. doi: 10.5772/61328

Chen, C. Y., Caporizzo, M. A., Bedi, K., Vite, A., Bogush, A. I., Robison, P., et al. (2018). Suppression of detyrosinated microtubules improves cardiomyocyte function in human heart failure. Nat. Med. 24, 1225-1233. doi: 10.1038/s41591018-0046-2

Chen, C.-H., Nakayama, M., Nevo, E., Fetics, B. J., Maughan, W. L., and Kass, D. A. (1998). Coupled systolic-ventricular and vascular stiffening with age. J. Am. Coll. Cardiol. 32, 1221-1227. doi: 10.1016/S0735-1097(98)00374-X

Chris, H., and Jacobs, J. (2017). Dissecting the role of the extracellular matrix in heart disease: lessons from the Drosophila genetic model. Vet. Sci. 4:24. doi: $10.3390 /$ vetsci4020024

\section{ETHICS STATEMENT}

The animal study was reviewed and approved by Institutional Animal Care and Use Committee.

\section{AUTHOR CONTRIBUTIONS}

MC and BP designed the experiments, analyzed the data, and wrote and edited the manuscript. MC conducted the experiments. Both authors contributed to the article and approved the submitted version.

\section{FUNDING}

This work was supported by funding from NHLBI R01-HL133080 and R01HL149891 to BP, NIAMS grant 5T32AR053461 to MC, and by the Center for Engineering MechanoBiology through NSF's Science and Technology Center program by NSF CMMI grant 15-48571. We acknowledge support from the Fondation Leducq Research Grant No. 20CVD01 to BP.

Christensen, G., Herum, K. M., and Lunde, I. G. (2019). Sweet, yet underappreciated: Proteoglycans and extracellular matrix remodeling in heart disease. Matrix Biol. 75-76, 286-299. doi: 10.1016/j.matbio.2018.01.001

Chung, C. S. (2020). Move quickly to detach: strain rate-dependent myosin detachment and cardiac relaxation. J. Gen. Physiol. 152:e202012588. doi: 10. 1085/jgp.202012588

Chung, C. S., Hoopes, C. W., and Campbell, K. S. (2017). Myocardial relaxation is accelerated by fast stretch, not reduced afterload. J. Mol. Cell Cardiol. 103, 65-73. doi: 10.1016/j.yjmcc.2017.01.004

Chung, C. S., Methawasin, M., Nelson, O. L., Radke, M. H., Hidalgo, C. G., Gotthardt, M., et al. (2011). Titin based viscosity in ventricular physiology: an integrative investigation of PEVK-actin interactions. J. Mol. Cell Cardiol. 7:5681.

Cooper, G. (2006). Cytoskeletal networks and the regulation of cardiac contractility: microtubules, hypertrophy, and cardiac dysfunction. Am. J. Physiol. Heart Circ. Physiol. 291, H1003-H1014. doi: 10.1152/ajpheart.00132. 2006

de Tombe, P. P., and ter Keurs, H. E. (1992). An internal viscous element limits unloaded velocity of sarcomere shortening in rat myocardium. J. Physiol. 454, 619-642. doi: 10.1113/jphysiol.1992.sp019283

Elwan, M. H., Roshdy, A., Reynolds, J. A., Elsharkawy, E. M., Eltahan, S. M., and Coats, T. J. (2018). What is the normal haemodynamic response to passive leg raise? A study of healthy volunteers. Emerg. Med. J. 35, 544-549. doi: 10.1136/ emermed-2017-206836

Falcão-Pires, I., Fontes-Sousa, A. P., Lopes-Conceição, L., Brás-Silva, C., and LeiteMoreira, A. F. (2011). Modulation of myocardial stiffness by $\beta$-adrenergic stimulation-its role in normal and failing heart. Physiol. Res. 31, 599-609. doi: $10.33549 /$ physiolres.932088

Fassett, J., Xu, X., Kwak, D., Zhu, G., Fassett, E. K., Zhang, P., et al. (2019). Adenosine kinase attenuates cardiomyocyte microtubule stabilization and protects against pressure overload-induced hypertrophy and LV dysfunction. J. Mol. Cell Cardiol. 130, 49-58. doi: 10.1016/j.yjmcc.2019.03.015

Fraites Thomas, J., Saeki, A., and Kass, D. A. (1997). Effect of altering filling pattern on diastolic pressure-volume curve. Circulation 96, 4408-4414. doi: 10.1161/01.CIR.96.12.4408

Frangogiannis, N. G. (2019). The extracellular matrix in ischemic and nonischemic heart failure. Circ. Res. 125, 117-146. doi: 10.1161/CIRCRESAHA.119.3 11148 
Fukuda, N., Wu, Y., Nair, P., and Granzier, H. L. (2005). Phosphorylation of titin modulates passive stiffness of cardiac muscle in a titin isoform-dependent manner. J. Gen. Physiol. 125, 257-271. doi: 10.1085/jgp.200409177

Grant, C. A., Phillips, M. A., and Thomson, N. H. (2012). Dynamic mechanical analysis of collagen fibrils at the nanoscale. J. Mech. Behav. Biomed. Mater. 5, 165-170. doi: 10.1016/j.jmbbm.2011.08.020

Granzier, H. L., and Irving, T. C. (1995). Passive tension in cardiac muscle: contribution of collagen, titin, microtubules, and intermediate filaments. Biophys. J. 68, 1027-1044. doi: 10.1016/S0006-3495(95)80278-X

Gulati, A., Jabbour, A., Ismail, T. F., Guha, K., Khwaja, J., Raza, S., et al. (2013). Association of fibrosis with mortality and sudden cardiac death in patients with nonischemic dilated cardiomyopathy. JAMA 309:896. doi: 10.1001/jama.2013. 1363

Hamdani, N., Bishu, K. G., von Frieling-Salewsky, M., Redfield, M. M., and Linke, W. A. (2013). Deranged myofilament phosphorylation and function in experimental heart failure with preserved ejection fraction. Cardiovasc. Res. 97, 464-471. doi: 10.1093/cvr/cvs353

Harris, T. S., Baicu, C. F., Conrad, C. H., Koide, M., Buckley, J. M., Barnes, M., et al. (2002). Constitutive properties of hypertrophied myocardium: cellular contribution to changes in myocardial stiffness. Am. J. Physiol. Heart Circ. Physiol. 282, H2173-H2182. doi: 10.1152/ajpheart.00480.2001

Helmes, M., Najafi, A., Palmer, B. M., Breel, E., Rijnveld, N., Iannuzzi, D., et al. (2016). Mimicking the cardiac cycle in intact cardiomyocytes using diastolic and systolic force clamps; measuring power output. Cardiovasc. Res. 111, 66-73. doi: $10.1093 / \mathrm{cvr} / \mathrm{cvw} 072$

Herwig, M., Kolijn, D., Lódi, M., Hölper, S., Kovács, Á, Papp, Z., et al. (2020). Modulation of titin-based stiffness in hypertrophic cardiomyopathy via protein kinase D. Front. Physiol. 11:240. doi: 10.3389/fphys.2020.00240

Hess, O. M., Grimm, J., and Krayenbuehl, H. P. (1979). Diastolic simple elastic and viscoelastic properties of the left ventricle in man. Circulation 59, 1178-1187. doi: 10.1161/01.CIR.59.6.1178

Hildick-Smith, D. J. R. (2001). Echocardiographic differentiation of pathological and physiological left ventricular hypertrophy. Heart 85, 615-619. doi: 10.1136/ heart.85.6.615

Humphrey, J. D., Strumpf, R. K., and Yin, F. C. (1990). Biaxial mechanical behavior of excised ventricular epicardium. Am. J. Physiol. 259, H101-H108. doi: 10. 1152/ajpheart.1990.259.1.H101

Kass, D. A. (2005). Ventricular arterial stiffening: integrating the pathophysiology. Hypertension 46, 185-193. doi: 10.1161/01.HYP.0000168053.34306.d4

Kass, D. A., Bronzwaer, J. G. F., and Paulus, W. J. (2004). What mechanisms underlie diastolic dysfunction in heart failure? Circ Res. 94, 1533-1542. doi: 10.1161/01.RES.0000129254.25507.d6

Kerr, J. P., Robison, P., Shi, G., Bogush, A. I., Kempema, A. M., Hexum, J. K., et al. (2015). Detyrosinated microtubules modulate mechanotransduction in heart and skeletal muscle. Nat. Commun. 6:8526. doi: $10.1038 /$ ncomms 9526

King, N. M. P., Methawasin, M., Nedrud, J., Harrell, N., Chung, C. S., Helmes, M., et al. (2011). Mouse intact cardiac myocyte mechanics: cross-bridge and titinbased stress in unactivated cells. J. Gen. Physiol. 137, 81-91. doi: 10.1085/jgp. 201010499

Koide, M., Hamawaki, M., Narishige, T., Nemoto, S., DeFreyte, G., Zile, M. R., et al. (2000). Microtubule depolymerization normalizes in vivo myocardial contractile function in dogs with pressure-overload left ventricular hypertrophy. Circulation 102, 1045-1052. doi: 10.1161/01.CIR.102.9.1045

Kovács, S. J., Meisner, J. S., and Yellin, E. L. (2000). Modeling of diastole. Cardiol. Clin. 18, 459-487. doi: 10.1016/s0733-8651(05)70156-9

Kuznetsov, A. V., Veksler, V., Gellerich, F. N., Saks, V., Margreiter, R., and Kunz, W. S. (2008). Analysis of mitochondrial function in situ in permeabilized muscle fibers, tissues and cells. Nat. Protoc. 3, 965-976. doi: 10.1038/nprot.2008. 61

Le Winter, M. M., and Van Buren, P. (2002). Myo?lament remodeling during the progression of heart failure. J. Card. Fail. 8(Suppl. 6), S271-S275. doi: 10.1054/ jcaf.2002.129276

LeWinter, M. M., and Palmer, B. M. (2015). Updating the physiology and pathophysiology of cardiac myosin-binding Protein-C. Circ. Heart Fail. 8, 417-421. doi: 10.1161/CIRCHEARTFAILURE.115.002146

Lieber, S. C., Aubry, N., Pain, J., Diaz, G., Kim, S.-J., and Vatner, S. F. (2004). Aging increases stiffness of cardiac myocytes measured by atomic force microscopy nanoindentation. Am. J. Physiol. Heart Circ. Physiol. 287, H645-H651. doi: 10.1152/ajpheart.00564.2003

Linke, W. A., and Fernandez, J. M. (2003). "Cardiac titin: molecular basis of elasticity and cellular contribution to elastic and viscous stiffness components in myocardium," in Mechanics of Elastic Biomolecules, eds W. A. Linke, H. Granzier, and M. S. Z. Kellermayer (Berlin: Springer), 483-497. doi: 10.1007/ 978-94-010-0147-2_9

Linke, W. A., and Leake, M. C. (2004). Multiple sources of passive stress relaxation in muscle fibres. Phys. Med. Biol. 49, 3613-3627. doi: 10.1088/0031-9155/49/16/ 009

Liu, W., and Wang, Z. (2019). Current understanding of the biomechanics of ventricular tissues in heart failure. Bioengineering 7:2. doi: 10.3390/ bioengineering7010002

Lyon, A. R., MacLeod, K. T., Zhang, Y., Garcia, E., Kanda, G. K., Lab, M. J., et al. (2009). Loss of T-tubules and other changes to surface topography in ventricular myocytes from failing human and rat heart. Proc. Natl. Acad. Sci. U.S.A. 106, 6854-6859. doi: 10.1073/pnas.0809777106

McDonald, K. S., and Moss, R. L. (1995). Osmotic compression of single cardiac myocytes eliminates the reduction in $\mathrm{Ca}^{2+}$ sensitivity of tension at short sarcomere length. Circ. Res. 77, 199-205. doi: 10.1161/01.RES.77.1.199

Miyata, S., Minobe, W., Bristow, M. R., and Leinwand, L. A. (2000). Myosin heavy chain isoform expression in the failing and nonfailing human heart. Circ. Res. 86, 386-390. doi: 10.1161/01.RES.86.4.386

Nagai, T., Honda, S., Sugano, Y., Matsuyama, T. A., Ohta-Ogo, K., Asaumi, Y., et al. (2014). Decreased myocardial dendritic cells is associated with impaired reparative fibrosis and development of cardiac rupture after myocardial infarction in humans. J. Am. Heart Assoc. 3:e000839. doi: 10.1161/JAHA.114. 000839

Nagueh, S. F. (2020). Left ventricular diastolic function. JACC Cardiovasc. Imag. 13, 228-244. doi: 10.1016/j.jcmg.2018.10.038

Nam, S., Hu, K. H., Butte, M. J., and Chaudhuri, O. (2016). Strain-enhanced stress relaxation impacts nonlinear elasticity in collagen gels. Proc. Natl. Acad. Sci. U.S.A. 113, 5492-5497. doi: 10.1073/pnas.1523906113

Nishimura, S., Nagai, S., Katoh, M., Yamashita, H., Saeki, Y., Okada, J., et al. (2006). Microtubules modulate the stiffness of cardiomyocytes against shear stress. Circ. Res. 98, 81-87. doi: 10.1161/01.RES.0000197785.51819.e8

Ogilvie, L. M., Edgett, B. A., Huber, J. S., Platt, M. J., Eberl, H. J., Lutchmedial, S., et al. (2020). Hemodynamic assessment of diastolic function for experimental models. Am. J. Physiol. Heart Circ. Physiol. 318, H1139-H1158. doi: 10.1152/ ajpheart.00705.2019

Palmer, B. M., Noguchi, T., Wang, Y., Heim, J. R., Alpert, N. R., Burgon, P. G., et al. (2004). Effect of cardiac myosin binding Protein-C on mechanoenergetics in mouse myocardium. Circ. Res. 94, 1615-1622. doi: 10.1161/01.RES.0000132744. 08754.f2

Perestrelo, A. R., Silva, A. C., Oliver-De La Cruz, J., Martino, F., Horváth, V., Caluori, G., et al. (2021). Multiscale analysis of extracellular matrix remodeling in the failing heart. Circ. Res. 128, 24-38. doi: 10.1161/CIRCRESAHA.120. 317685

Rankin, J. S., Arentzen, C. E., McHale, P. A., Ling, D., and Anderson, R. W. (1977). Viscoelastic properties of the diastolic left ventricle in the conscious dog. Circ. Res. 41, 37-45. doi: 10.1161/01.RES.41.1.37

Robison, P., Caporizzo, M. A., Ahmadzadeh, H., Bogush, A. I., Chen, C. Y., Margulies, K. B., et al. (2016). Detyrosinated microtubules buckle and bear load in contracting cardiomyocytes. Science 352:aaf0659. doi: 10.1126/science. aaf0659

Rosas, P. C., Liu, Y., Abdalla, M. I., Thomas, C. M., Kidwell, D. T., Dusio, G. F., et al. (2015). Phosphorylation of cardiac myosin-binding Protein-C is a critical mediator of diastolic function. Circ. Heart Fail. 8, 582-594. doi: 10.1161/ CIRCHEARTFAILURE.114.001550

Sequeira, V., Nijenkamp, L. L. A. M., Regan, J. A., and van der Velden, J. (2014). The physiological role of cardiac cytoskeleton and its alterations in heart failure. Biochim. Biophys. Acta BBA Biomembr. 1838, 700-722. doi: 10.1016/j.bbamem. 2013.07.011

Smiseth, O. A. (2018). Evaluation of left ventricular diastolic function: state of the art after 35 years with doppler assessment. J. Echocardiogr. 16, 55-64.

Sonnenblick, E. H. (1962). Force-velocity relations in mammalian heart muscle. Am. J. Physiol. Leg. Content 202, 931-939. doi: 10.1152/ajplegacy.1962. 202.5.931 
Sonnenblick, E. H., Ross, J., Covell, J. W., Spotnitz, H. M., and Spiro, D. (1967). The ultrastructure of the heart in systole and diastole: changes in sarcomere length. Circ. Res. 21, 423-431. doi: 10.1161/01.RES.21.4.423

Stroud, J. D., Baicu, C. F., Barnes, M. A., Spinale, F. G., and Zile, M. R. (2002). Viscoelastic properties of pressure overload hypertrophied myocardium: effect of serine protease treatment. Am. J. Physiol. Heart Circ. Physiol. 282, H2324H2335. doi: 10.1152/ajpheart.00711.2001

Swiatlowska, P., Sanchez-Alonso, J. L., Wright, P. T., Novak, P., and Gorelik, J. (2020). Microtubules regulate cardiomyocyte transversal Young's modulus. Proc. Natl. Acad. Sci. U.S.A. 117, 2764-2766. doi: 10.1073/pnas.1917171117

Templeton, G. H., Wildenthal, K., Willerson, J. T., and Reardon, W. C. (1974). Influence of temperature on the mechanical properties of cardiac muscle. Circ. Res. 34, 624-634. doi: 10.1161/01.RES.34.5.624

Tsutsui, H., Ishihara, K., and Cooper, G. (1993). Cytoskeletal role in the contractile dysfunction of hypertrophied myocardium. Science 260, 682-687. doi: 10.1126/ science. 8097594

van der Velden, J. (2011). Diastolic myofilament dysfunction in the failing human heart. Pflüg Arch. Eur. J. Physiol. 462, 155-163. doi: 10.1007/s00424-011-09603

van Heerebeek, L., Hamdani, N., Handoko, M. L., Falcao-Pires, I., Musters, R. J., Kupreishvili, K., et al. (2008). Diastolic stiffness of the failing diabetic heart: importance of fibrosis, advanced glycation end products, and myocyte resting tension. Circulation 117, 43-51. doi: 10.1161/CIRCULATIONAHA.107.728550

Wang, Z., Golob, M. J., and Chesler, N. C. (2016). "Viscoelastic properties of cardiovascular tissues," in Viscoelastic and Viscoplastic Materials, ed. M. F. El-Amin (London: InTech Open), doi: 10.5772/64169

Webber, M., Jackson, S. P., Moon, J. C., and Captur, G. (2020). Myocardial Fibrosis in heart failure: anti-fibrotic therapies and the role of cardiovascular magnetic resonance in drug trials. Cardiol. Ther. 9, 363-376. doi: 10.1007/s40119-02000199-y

Yellin, E. L., Meisner, J. S., Nikolic, S. D., and Keren, G. (1992). The scientific basis for the relations between pulsed-doppler transmitral velocity patterns and left heart chamber properties. Echocardiography 9, 313-338. doi: 10.1111/j.15408175.1992.tb00474.x

Zhang, W., and Kovács, S. J. (2008). The diastatic pressure-volume relationship is not the same as the end-diastolic pressure-volume relationship. Am. J. Physiol. Heart Circ. Physiol. 294, H2750-H2760. doi: 10.1152/ajpheart.00200. 2008

Zile, M. R., and Brutsaert, D. L. (2002). New concepts in diastolic dysfunction and diastolic heart failure: part I: diagnosis, prognosis, and measurements of diastolic function. Circulation 105, 1387-1393. doi: 10.1161/hc1102.1 05289

Zile, M. R., Baicu, C. F., Ikonomidis, S., Stroud, R. E., Nietert, P. J., Bradshaw, A. D., et al. (2015). Myocardial stiffness in patients with heart failure and a preserved ejection fraction: contributions of collagen and titin. Circulation 131, 1247-1259. doi: 10.1161/CIRCULATIONAHA.114.013215

Conflict of Interest: The authors declare that the research was conducted in the absence of any commercial or financial relationships that could be construed as a potential conflict of interest.

Publisher's Note: All claims expressed in this article are solely those of the authors and do not necessarily represent those of their affiliated organizations, or those of the publisher, the editors and the reviewers. Any product that may be evaluated in this article, or claim that may be made by its manufacturer, is not guaranteed or endorsed by the publisher.

Copyright (c) 2021 Caporizzo and Prosser. This is an open-access article distributed under the terms of the Creative Commons Attribution License (CC BY). The use, distribution or reproduction in other forums is permitted, provided the original author(s) and the copyright owner(s) are credited and that the original publication in this journal is cited, in accordance with accepted academic practice. No use, distribution or reproduction is permitted which does not comply with these terms. 